\title{
Exposure to Emerging and Legacy Polyfluoroalkyl Substances in the Sixth Total Diet Study — China, 2016-2019
}

\author{
Yuxin Wang'; Xueyan $\mathrm{Gao}^{2}$; Jiaying $\mathrm{Liu}^{2, *} ;$ Bing $\mathrm{Lyu}^{1, * ;}$; Jingguang $\mathrm{Li}^{1}$; Yunfeng Zhao'; Yongning $\mathrm{Wu}^{1}$
}

\section{Summary \\ What is already known about this topic? \\ Perfluoroalkyl substances (PFASs) are persistent organic pollutants, which have multi-organ toxicity and potential health risk to humans. \\ What is added by this report? \\ The most commonly detected PFASs in the Sixth China Total Diet Study (TDS) samples were perfluorooctanesulfonate (PFOS), perfluorooctanoic acid (PFOA), perfluoroundecanoic acid (PFUdA), perfluorodecanoic acid (PFDA), perfluorononanoic acid (PFNA), and 9-chlorohexadecafluoro-3- oxanonane-1-sulfonate (6:2 Cl-PFESA). The mean estimated weekly intakes (EWIs) of PFOA, PFOS, and 6:2 Cl-PFESA in the Sixth TDS were 2.17, 2.72, and $2.75 \mathrm{ng} / \mathrm{kg}$ body weight per week, respectively. \\ What are the implications for public health practice? \\ The PFASs levels in some food category and dietary exposure still need to be continuously monitored, especially for 6:2 Cl-PFESA.}

Perfluoroalkyl substances (PFASs) are persistent organic pollutants, which are widely used in consumer and industrial products and can cause direct or indirect toxic effects on multiple organs (1-2). Due to the increasingly stringent controls on the production and use of perfluorooctanoic acid (PFOA) and perfluorooctanesulfonate (PFOS), plenty of alternatives are used. However, recent studies indicated that some alternatives may be more bio-accumulative or toxic than those legacy PFASs (3-4). In order to investigate the latest dietary exposure of legacy and emerging contaminants for Chinese adults, composite food samples were collected from 24 provincial-level administrative divisions (PLADs) in the Sixth China Total Diet Study (TDS) during 2016-2019. The mean EWIs of PFOS, PFOA, and 9chlorohexadecafluoro-3-oxanonane-1-sulfonate (6:2 Cl-PFESA) in the Sixth TDS were 2.72, 2.17, and
$2.75 \mathrm{ng} / \mathrm{kg}$ body weight per week, respectively, indicating low health risks via dietary exposure of these PFASs for most of the population in China. The related results could provide basic data for the health effect assessment of PFASs and also provide a scientific basis for control measures of health damage caused by PFASs.

In this study, 25 legacy PFASs including typical isomers [perfluorohexanoic acid (PFHxA), perfluoroheptanoic acid (PFHpA), perfluoro-noctanoic acid (n-PFOA), perfluoro-4-methylheptanoic acid (4m-PFOA), perfluoro-5-methylheptanoic acid (5m-PFOA), perfluoro-6-methylheptanoic acid (isoPFOA), perfluorononanoic acid (PFNA), perfluorodecanoic acid (PFDA), perfluoroundecanoic acid (PFUdA), perfluorododecanoic acid (PFDoA), perfluorotridecanoic acid (PFTrDA), perfluorotetradecanoic acid (PFTeDA), perfluorobutanesulfonate (PFBS), perfluoropentanesulfonate (PFPeS), perfluorohexanesulfonate (PFHxS), perfluoroheptanesulfonate (PFHpS), perfluoro-1-octanesulfonate (n-PFOS), perfluoro-1-methylheptanesulfonate (1mPFOS), perfluoro-3-methylheptanesulfonate (3mPFOS), perfluoro-4-methylheptanesulfonate (4mPFOS), perfluoro-5-methylheptanesulfonate (5mPFOS), and perfluoro-6-methylheptanesulfonate (isoPFOS), perfluorononanesulfonate (PFNS), perfluorodecanesulfonate (PFDS), and perfluorododecanesulfonate (PFDoS)] and 3 emerging PFASs [6:2 Cl-PFESA, 11-chloroeicosafluoro-3oxaundecane-1-sulfonate (8:2 Cl-PFESA), and dodecafluoro-3H-4,8-dioxanonanoate (ADONA)] were analyzed in the food samples. The details of the TDS are described in the Foreword of this issue (5). The sample preparation and instrumental analysis was described in detail in our previous studies $(6-7)$. The concentrations below the limits of detection (LOD) were set to be LOD/2.

In the present study, PFHxA, PFHpS, PFNS, PFDS, PFDoS, 8:2 Cl-PFESA, and ADONA were not found in all food samples $(n=216)$. The summary of detection frequency (DF) and concentration of 
detectable PFASs and alternatives in all food samples are shown in Supplementary Table S1 (available in https://weekly.chinacdc.cn/). The most commonly detected PFASs in all samples were PFOS, PFOA, PFUdA, PFDA, PFNA and 6:2 Cl-PFESA, the last of which was one of the contaminants of high concern in recent years. Generally, the DFs of perfluoroalkyl carboxylates (PFCAs) were higher than that of the PFSAs. Except for milk samples, the DF of PFASs in animal-origin food samples was much higher than that in vegetal-origin food samples. None of PFASs and their alternatives were detected in the milk samples. Most of the PFASs were detected in aquatic food, especially for long chain PFCAs, PFOS, and its predominant alternative (6:2 Cl-PFESA) with $100 \%$ DF. Similar to the DF, the levels of PFASs in foods of animal-origin were much higher than those vegetalorigin food samples. The estimated weekly intakes (EWIs) of PFOS, PFOA, and 6:2 Cl-PFESA in 24 PLADs were shown in Table 1 and Figure 1. The mean EWIs of PFOS and PFOA in the Sixth TDS accounted for $20.9 \%$ and $36.2 \%$ of the tolerable weekly intake (TWI) set by EFSA in $2018(13 \mathrm{ng} / \mathrm{kg}$ body weight per week for PFOS and $6 \mathrm{ng} / \mathrm{kg}$ body weight per week for PFOA) (8). The average TWI of 6:2 Cl-PFESA was very close to PFOS.

\section{DISCUSSION}

In the present study, contamination of PFCAs was found commonly in aquatic food. Most of long chain PFCAs (C9-C14) were detected with higher DF and occurrence levels than those of PFOA in aquatic food samples. However, PFOA was still the predominant PFACs in other food groups both for DF and levels. By median, the highest PFOA level was found in the meats group, followed by aquatic food and eggs group. In the meats group, the PFOA level from Shandong was the highest, followed by Zhejiang. Moreover, for the levels of PFOA in aquatic food and eggs group, the highest two values were both from Beijing Municipality and Shandong Province, indicating more PFOA contamination in Shandong than in other PLADs involved in the Sixth China TDS. In vegetalorigin foods, PFOA was only detected in scattered samples with very low levels. Except for PFTeDA, other long chain PFCAs (PFNA, PFDA, PFUdA, PFDoA, and PFTrDA) were found in the meats group, eggs group, and aquatic food, but the DFs and levels of these PFCAs were significantly lower than those in aquatic food. In addition, main isomers of PFOA were
TABLE 1. The EWls (ng/kg body weight per week) of PFOA, PFOS, and 6:2 Cl-PFESA in 24 PLADs in China, 2016-2019.

\begin{tabular}{|c|c|c|c|}
\hline PLADs & PFOA & PFOS & 6:2 Cl-PFESA \\
\hline Heilongjiang & 0.77 & 14.28 & 1.03 \\
\hline Liaoning & 0.67 & 1.46 & 0.30 \\
\hline Hebei & 0.61 & 0.96 & 0.10 \\
\hline Beijing & 13.73 & 1.59 & 0.15 \\
\hline Jilin & 0.70 & 1.88 & 0.34 \\
\hline Shanxi & 0.70 & 1.62 & 0.30 \\
\hline Shaanxi & 8.54 & 0.88 & 0.05 \\
\hline Henan & 0.27 & 0.76 & 0.20 \\
\hline Ningxia & 2.61 & 0.43 & 0.02 \\
\hline Inner Mongolia & 0.89 & 1.04 & 0.27 \\
\hline Qinghai & 0.32 & 0.41 & 0.03 \\
\hline Gansu & 0.61 & 0.57 & 0.05 \\
\hline Shanghai & 3.52 & 5.23 & 3.94 \\
\hline Fujian & 1.90 & 2.32 & 0.55 \\
\hline Jiangxi & 2.73 & 4.15 & 0.44 \\
\hline Jiangsu & 2.07 & 2.65 & 1.91 \\
\hline Zhejiang & 3.74 & 8.67 & 51.57 \\
\hline Shandong & 5.32 & 2.31 & 0.38 \\
\hline Hubei & 0.63 & 2.43 & 0.54 \\
\hline Sichuan & 0.58 & 1.63 & 0.59 \\
\hline Guangxi & 0.28 & 1.17 & 0.13 \\
\hline Hunan & 0.33 & 5.37 & 2.63 \\
\hline Guangdong & 0.18 & 2.84 & 0.47 \\
\hline Guizhou & 0.42 & 0.55 & 0.04 \\
\hline Average & 2.17 & 2.72 & 2.75 \\
\hline
\end{tabular}

Abbreviations: PFOS=perfluorooctanesulfonate; $\mathrm{PFOA}=$ perfluorooctanoic acid; 6:2 Cl-PFESA=9-chlorohexadecafluoro-3oxanonane-1-sulfonate; PLADs=provincial-level administrative divisions.

analyzed in all food samples. However, br-PFOA was only found in the egg sample from Beijing Municipality with a contribution of $5.6 \%$ to total PFOA levels in that sample.

For PFSAs, unlike the case of PFCAs, only PFOS were commonly found in animal-origin food. In general, the DF and level of PFOS was more than that of PFOA in aquatic food and egg groups, with the highest median concentration of $0.29 \mathrm{ng} / \mathrm{g}$ wet weight in aquatic food. The highest PFOS levels in aquatic food were found in Heilongjiang, which has been higher than the highest PFOS levels in aquatic food in the fourth and fifth TDS $(3.47 \mathrm{ng} / \mathrm{g}$ wet weight and $1.65 \mathrm{ng} / \mathrm{g}$ wet weight, respectively) (9). Some coastal PLADs also showed higher pollution levels of PFOS, 


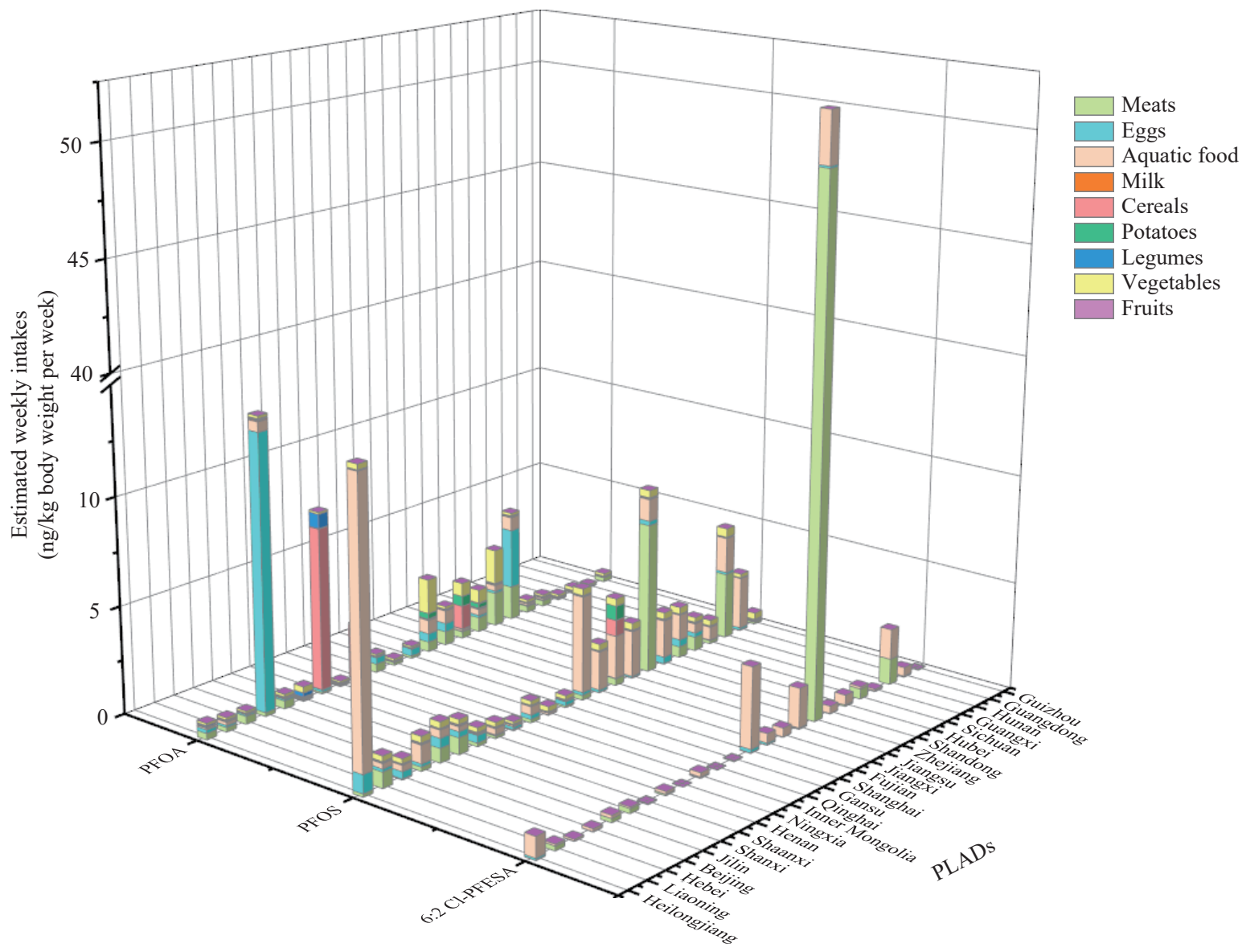

FIGURE 1. The estimated weekly intakes of PFOS, PFOA, and 6:2 CI-PFESA in 24 PLADs of China, 2016-2019.

Abbreviations: PFOS=perfluorooctanesulfonate; PFOA=perfluorooctanoic acid; 6:2 Cl-PFESA=9-chlorohexadecafluoro-3oxanonane-1-sulfonate; PLADs=provincial-level administrative divisions.

such as Shandong, Shanghai, and Jiangsu. In the eggs group, the highest PFOS was also detected in Heilongjiang, followed by Shaanxi and Sichuan. By median, PFOS level in meats group was lower than that in eggs group. In contrast to PFOA, br-PFOS were commonly detected in aquatic food, which accounted for $3.3 \%-21.2 \%$ of total PFOS. The proportions of br-PFOS to total PFOS were all much lower than those in the industrial products. These results may be related to the different bioaccumulation behavior of PFOS isomers in different animal species. Although PFBS, PFPeS, and PFHxS were rarely detected in the present study, PFPeS was found in some vegetable samples with a DF of $37.5 \%$, which was similar to another study, in which short-chain compounds had been found to accumulate at high levels in leafy vegetables (10).

This was the first time to determine the contamination of main alternatives of PFOA and PFOS in China TDS. 6:2 Cl-PFESA, one of the important alternatives of PFOS in China, was detected in all aquatic food samples with the highest median level among all food groups. The highest $6: 2 \mathrm{Cl}$ PFESA in aquatic food samples was observed in Shanghai, which was close to the level of PFOS in that sample. Moreover, the level of 6:2 Cl-PFESA in meats from Zhejiang was the highest value $(3.74 \mathrm{ng} / \mathrm{g}$ wet weight) in all samples, which was also much higher than the level of PFOS $(0.53 \mathrm{ng} / \mathrm{g}$ wet weight) in the same sample. The results indicated more attention should be paid to this emerging contaminant in food.

Unlike previous studies, the contribution of vegetalorigin food consumption to PFASs exposure was also considered in the Sixth Chinese Total Diet Study. Although the levels of PFOA in vegetal-origin food were much lower than those in animal-origin food, the high consumptions of vegetal-origin food led to their significant contribution to dietary exposure in some PLADs. As shown in Table 1, the highest EWI of PFOA was found in Beijing, followed by Shaanxi and Shandong. The EWIs of PFOA in Beijing and Shaanxi have exceeded the recommended value established by 
the EFSA in 2018. By average, eggs and meat groups were the primary source of PFOA in China, which was different with the survey results in EU by EFSA, in which fish and other seafood were the most important contributors to PFOA. The highest EWI of PFOS was found in Heilongjiang, followed by Zhejiang and Hunan. Only the EWI of PFOS in Heilongjiang exceeded the recommended value established by EFSA in 2018. On average, aquatic food and meats group were the primary source of PFOS, which was similar to the fourth and fifth China TDS, as well as the survey results of EFSA.

The highest EWI of 6:2 Cl-PFESA was observed in Zhejiang, followed by Shanghai and Hunan. On average, the meats group was the main source of $6: 2$ Cl-PFESA. It should be noted that, although there is no health-based guide value for 6:2 Cl-PFESA, the EWI of 6:2 Cl-PFESA in Zhejiang has been almost 4 times higher than TWI of PFOS established by EFSA. Therefore, the potential health risk of 6:2 Cl-PFESA should not be ignored considering it might be the most bio-persistent PFAS (4).

In general, the present study indicated low health risks via dietary exposure of some PFASs for most of the population in China. However, the levels in food and dietary exposure of high concern PFASs still need to be continuously monitored, which was also an urgent step to address the gap for the corresponding food safety policy in China. In addition, our results only showed the average dietary exposure of each province. For the dietary exposure of individual in different gender and age, probabilistic estimate should be developed. Monitoring of PFASs in food and establishing the maximum limit of PFASs could effectively reduce the health risk of dietary exposure.

Acknowledgements: The colleagues of 24 provincial CDCs.

Conflicts of interest: No conflicts of interest.

Funding: Supported by the National Key Research and Development Program of China (grant number 2017YFC1600500) and CAMS Innovation Fund for Medical Science (CIFMS 2019-I2M-5-024).

doi: $10.46234 / \mathrm{ccdcw} 2022.042$
\# Corresponding authors: Jiaying Liu, liujiaying@cau.edu.cn; Bing Lyu, lvbing@cfsa.net.cn.

\begin{abstract}
NHC Key Laboratory of Food Safety Risk Assessment, Chinese Academy of Medical Science Research Unit (No. 2019RU014), China National Center for Food Safety Risk Assessment, Beijing, China; 2 Key Laboratory of Precision Nutrition and Food Quality, Department of Nutrition and Health, China Agricultural University, Beijing, China.
\end{abstract}

Submitted: November 18, 2021; Accepted: February 28, 2022

\section{REFERENCES}

1. Olsen GW, Burris JM, Ehresman DJ, Froehlich JW, Seacat AM, Butenhoff JL, et al. Half-life of serum elimination of perfluorooctanesulfonate, perfluorohexanesulfonate, and perfluorooctanoate in retired fluorochemical production workers. Environ Health Perspect 2007;115(9):1298 - 305. http://dx.doi.org/ 10.1289/ehp.10009.

2. Steenland K, Fletcher T, Savitz DA. Epidemiologic evidence on the health effects of perfluorooctanoic acid (PFOA). Environ Health Perspect 2010;118(8):1100 - 8. http://dx.doi.org/10.1289/ehp. 0901827.

3. Cui QQ, Pan YT, Zhang HX, Sheng N, Dai JY. Elevated concentrations of perfluorohexanesulfonate and other per- and polyfluoroalkyl substances in Baiyangdian Lake (China): source characterization and exposure assessment. Environ Pollut 2018;241: 684 - 91. http://dx.doi.org/10.1016/j.envpol.2018.05.099.

4. Shi YL, Vestergren R, Xu L, Zhou Z, Li CX, Liang Y, et al. Human exposure and elimination kinetics of chlorinated polyfluoroalkyl ether sulfonic acids (Cl-PFESAs). Environ Sci Technol 2016;50(5):2396 404. http://dx.doi.org/10.1021/acs.est.5b05849.

5. Lyu B, Li JG, Wu YN. Characterizing exposome of food contamination and China Total Diet Study: project for improving food safety risk assessment in China. China CDC Wkly 2022; 4(9): 157-60.

6. Wang XP, Wang YX, Li JG, Liu JF, Zhao YF, Wu YN. Occurrence and dietary intake of perfluoroalkyl substances in foods of the residents in Beijing, China. Food Addit Contam: Part B Surveill 2021;14(1):1 - 11. http://dx.doi.org/10.1080/19393210.2020.1821098.

7. Wang YX, Zhang L, Teng Y, Zhang JY, Yang L, Li JG, et al. Association of serum levels of perfluoroalkyl substances with gestational diabetes mellitus and postpartum blood glucose. J Environ Sci 2018;69:5 - 11. http://dx.doi.org/10.1016/j.jes.2018.03.016.

8. Knutsen HK, Alexander J, Barregård L, Bignami M, Brüschweiler B, Ceccatelli $S$, et al. Risk to human health related to the presence of perfluorooctane sulfonic acid and perfluorooctanoic acid in food. EFSA J 2018;16(12):e05194. http://dx.doi.org/10.2903/j.efsa.2018.5194.

9. Wang YX, Liu JY, Li JG, Zhao YF, Wu YN. Dietary exposure of Chinese adults to perfluoroalkyl acids via animal-origin foods: Chinese total diet study (2005-2007 and 2011-2013). J Agric Food Chem 2019;67(21):6048 - 55. http://dx.doi.org/10.1021/acs.jafc.9b01108.

10. Ghisi R, Vamerali T, Manzetti S. Accumulation of perfluorinated alkyl substances (PFAS) in agricultural plants: a review. Environ Res 2019;169:326 - 41. http://dx.doi.org/10.1016/j.envres.2018.10.023. 


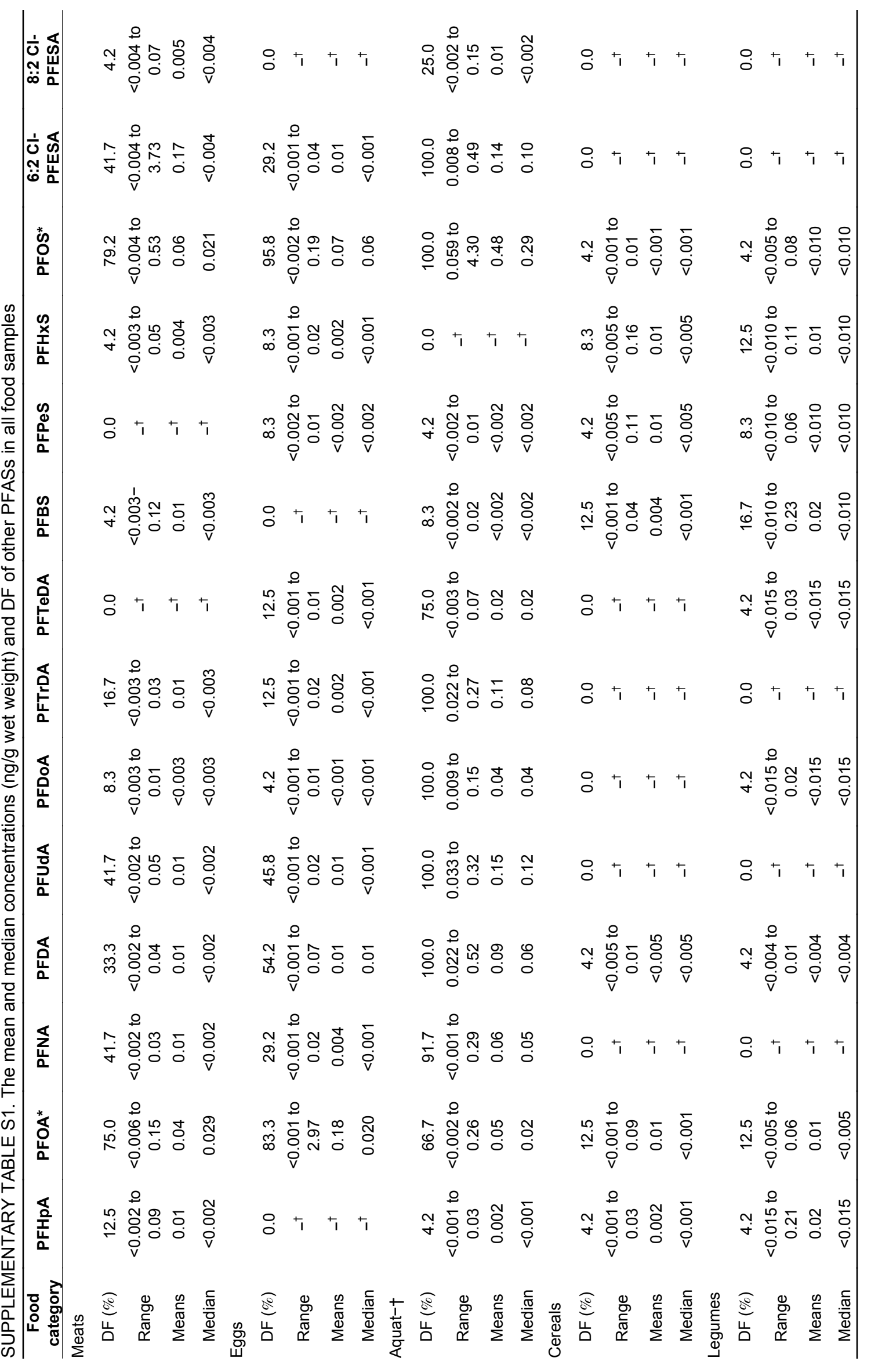




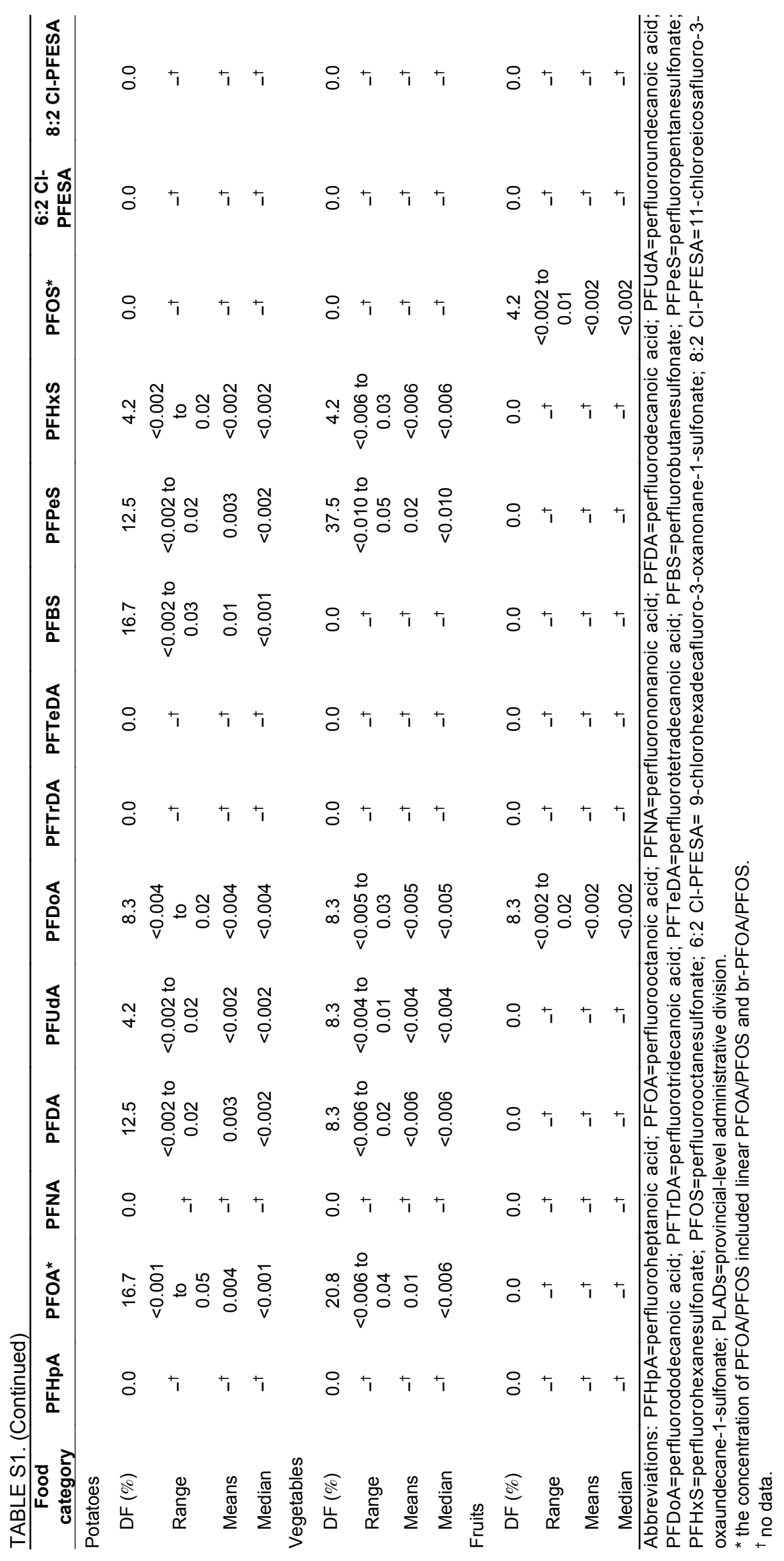

